\title{
Observation of Intersubband Absorption in ZnO Coupled Quantum Wells
}

\author{
Bo Meng® ${ }^{1,}{ }^{*}$ Julen Tamayo-Arriola, ${ }^{2}$ Nolwenn Le Biavan, ${ }^{3}$ Miguel Montes Bajo, ${ }^{2}$ \\ Almudena Torres-Pardo, ${ }^{4}$ Maxime Hugues, ${ }^{3}$ Denis Lefebvre, ${ }^{3}$ Adrian Hierro, ${ }^{2}$ \\ Jean-Michel Chauveau $\odot,{ }^{3}$ and Jérôme Faist ${ }^{1}$ \\ ${ }^{1}$ Institute for Quantum Electronics, ETH Zurich, 8093 Zürich, Switzerland \\ ${ }_{3}^{2}$ ISOM, Universidad Politécnica de Madrid, 28040 Madrid, Spain \\ ${ }^{3}$ Université Côte d'Azur, CNRS, CRHEA, 06560 Valbonne, France \\ ${ }^{4}$ Departamento de Química Inorgánica, Universidad Complutense de Madrid, 28040 Madrid, Spain
}

(Received 22 July 2019; revised manuscript received 13 September 2019; published 4 November 2019)

\begin{abstract}
Intersubband transitions in $\mathrm{ZnO}$ material systems are predicted to be promising candidates for infrared and terahertz $(\mathrm{THz})$ optoelectronic devices due to their unusual material properties. In particular, the temperature performance of $\mathrm{THz}$ quantum cascade lasers is postulated to be significantly enhanced using $\mathrm{ZnO}$ material systems due to their large optical phonon energy. Taking a step forward toward that goal, intersubband transitions in $\mathrm{ZnO} / \mathrm{Mg}_{x} \mathrm{Zn}_{1-x} \mathrm{O}$ asymmetric coupled quantum wells are observed on a nonpolar $m$ plane $\mathrm{ZnO}$ substrate. Two absorption peaks are observed in the energy range from approximately 250 $\mathrm{meV}$ to approximately $410 \mathrm{meV}$ at room temperature, unambiguously demonstrating the interwell coupling in the asymmetric coupled quantum wells. A theoretical model taking into account the interaction between intersubband transitions shows reasonable overall agreement with the experimental results, thus proving the strong coupling nature of the investigated system. As the building block of complex quantum structures based on intersubband transitions, the results presented show great potential applications of $\mathrm{ZnO} / \mathrm{Mg}_{x} \mathrm{Zn}_{1-x} \mathrm{O}$ material systems in infrared and $\mathrm{THz}$ optoelectronics and physics.
\end{abstract}

DOI: 10.1103/PhysRevApplied.12.054007

\section{INTRODUCTION}

Zinc oxide $(\mathrm{ZnO})$ is a direct band-gap semiconductor material, which has been widely investigated thanks to its favorable physical and chemical properties. For instance, the large band-gap $(3.37 \mathrm{eV})$ and exciton binding energies allow the realization of efficient excitonic nature devices such as light-emission diodes (LEDs) [1-3] and lasers at ultraviolet (UV) range [4-6]. In fundamental physics, both the quantum Hall effect (QHE) [7] and the fractional quantum Hall effect have been observed [8,9]. The latter can only be explained by the strongly correlated carriers driven by the strong Coulomb interaction, which is significantly enhanced by the large effective mass $\left(0.24 m_{0}\right.$, where $m_{0}$ is the free electron mass) of the electrons in the $\mathrm{ZnO}$ material system [10]. While being extensively studied, an underexplored yet extremely interesting property of $\mathrm{ZnO}$ is its large optical phonon energy (approximately $72 \mathrm{meV}$ )

*bmeng@phys.ethz.ch

Published by the American Physical Society under the terms of the Creative Commons Attribution 4.0 International license. Further distribution of this work must maintain attribution to the author(s) and the published article's title, journal citation, and DOI. compared with those of GaAs and (In, Ga)As commonly used for the intersubband optoelectronic devices. Even though the $p$ doping of $\mathrm{ZnO}$ remains a huge challenge, it does not hinder $\mathrm{ZnO}$ from being a candidate for unipolar devices based on intersubband transitions (ISBTs), such as quantum cascade detectors (QCDs) [11,12], phononpolariton lasers [13], and quantum cascade lasers (QCLs) [14], especially in the THz range at high temperature [15]. In fact, for ISBTs below the optical phonon energy of $\mathrm{ZnO}$ [e.g., in the terahertz $(\mathrm{THz})$ range], the population inversion of THz QCLs is significantly enhanced at high temperature due to the suppressed optical phonon emission rate, thus significantly enhancing the high temperature operation of THz QCLs [16,17]. However, to achieve that goal requires excellent growth quality of the material system, such as the accurate control of the layer thickness over the entire active region, the sharp heterointerfaces between different layers to reduce the detrimental effect of interface roughness, the constant doping level in all periods, and so on. $[18,19]$.

Compared with quantum wells (QWs) grown on a polar substrate, the heterostructures grown on the nonpolar $\mathrm{ZnO}$ substrate are preferable as the quantum confined Stark effect (QCSE) is significantly suppressed, thus increasing the oscillator strength of the ISBTs [20]. The 
(a)

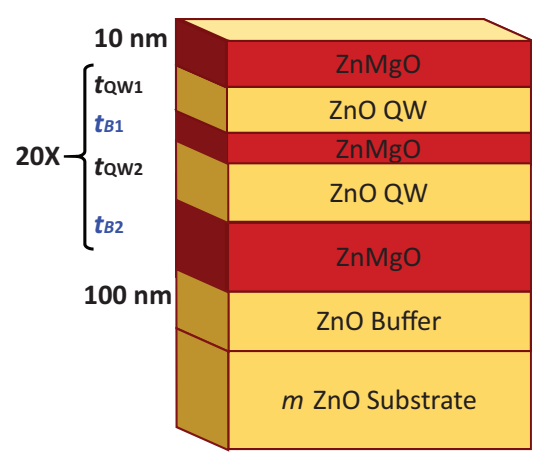

(b)

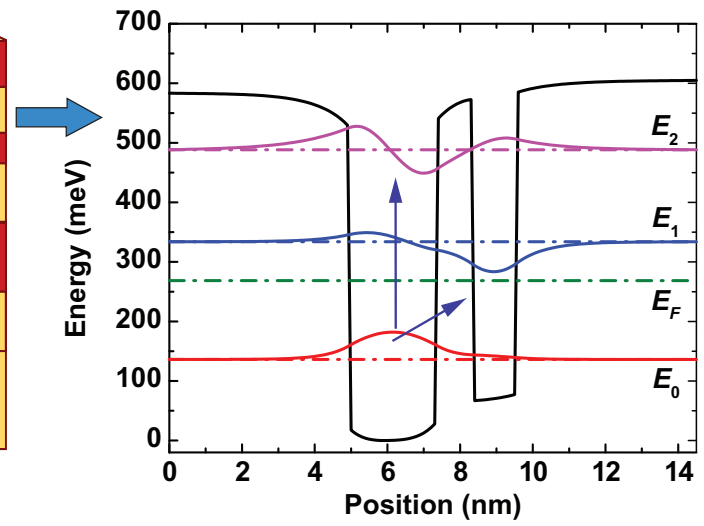

FIG. 1. Structures of the ACQWs samples investigated in the work. (a) Schematic of the waveguide structure for all the samples. (b) Calculated conduction band structure and wave functions for sample with $1-\mathrm{nm}$ thick barrier at room temperature $(300 \mathrm{~K})$. The main intersubband transitions are labeled with blue arrows. development of commercially available nonpolar, native $\mathrm{ZnO}$ substrates and excellent growth control has led to the first observation of the intersubband absorption for transverse magnetic (TM) polarization in multiple QWs grown on the $m$ plane substrate [21]. However, for further applications, many devices based on intersubband transitions require active structures beyond the single QW. Therefore, detailed optical study of a pair of asymmetric coupled quantum wells (ACQWs) [22], as the minimal building block for QCLs and QCDs, is necessary. In this work, we report the observation of intersubband absorption indicating strong electron coupling in ACQWs based on a $\mathrm{ZnO} / \mathrm{Mg}_{x} \mathrm{Zn}_{1-x} \mathrm{O}$ material system grown on a nonpolar $m$ plane $\mathrm{ZnO}$ substrate, which is a step forward toward the realization of THz QCLs based on a $\mathrm{ZnO} / \mathrm{Mg}_{x} \mathrm{Zn}_{1-x} \mathrm{O}$ system.

\section{DESIGN AND STRUCTURAL CHARACTERIZATION}

The structure of multi-ACQWs studied in this work, grown by the molecular beam epitaxy (MBE) on $m$ plane substrates, is shown in Fig. 1(a). Three $\mathrm{ZnO} / \mathrm{Mg}_{x} Z_{n_{1-x} \mathrm{O}}$ multi-ACQW samples with different $\mathrm{Mg}_{x} \mathrm{Zn}_{1-x} \mathrm{O}$ barrier thicknesses separating the coupled $\mathrm{ZnO}$ QWs are grown. Detailed sample structures are shown in Table I. The active

TABLE I. Thickness in $\mathrm{nm}$ of the thin well, the large well, the barrier separating both QWs, and the barrier separating each period for samples $A-C$. The thicknesses in the brackets are the measured values deduced from the $\mathrm{x}$-ray reflectivity and scanning electron microscopy measurements.

\begin{tabular}{lllll}
\hline \hline Sample & $t_{\mathrm{QW} 1}(\mathrm{~nm})$ & $t_{\mathrm{QW} 2}(\mathrm{~nm})$ & $t_{B 1}(\mathrm{~nm})$ & $t_{B 2}(\mathrm{~nm})$ \\
\hline$A$ & $1.2(1.3)$ & $2.5(2.7)$ & $0.5(0.5)$ & $10(10.0)$ \\
$B$ & $1.2(1.3)$ & $2.5(2.7)$ & $1.0(1.0)$ & $10(9.9)$ \\
$C$ & $1.2(1.3)$ & $2.5(2.7)$ & $1.5(1.5)$ & $10(9.9)$ \\
\hline \hline
\end{tabular}

regions of all samples consist of 20 periods of ACQWs to enhance the intersubband absorption. The wide QWs are uniformly Ga-doped to $n \simeq 5 \times 10^{19} \mathrm{~cm}^{-3}$ for all samples to populate the ground energy level. A $100-\mathrm{nm}$-thick undoped $\mathrm{ZnO}$ buffer is grown before the active region and the growth is finished with a $10-\mathrm{nm}$-undoped $\mathrm{Mg}_{x} Z_{\mathrm{n}_{1-x} \mathrm{O}} \mathrm{O}$ capping layer. The $\mathrm{Mg}$ content (30\%) is kept constant for all the samples. Detailed MBE growth conditions can be found in Ref. [18]. The conduction band diagram of one of the ACQWs with a 1-nm-thick $\mathrm{Mg}_{0.3} \mathrm{Zn}_{0.7} \mathrm{O}$ barrier is shown in Fig. 1(b), with arrows indicating the ISBTs. To model the ISBTs in ACQWs, a Schrödinger-Poisson solver, including the exchange-correlation effect [23] due to high doping level, is self-consistently solved to calculate the transitions in order to take into account the band structure modifications due to the inhomogeneous carrier distribution within the structure. For the parameter used, following Ref. [21], the band offset between the QW and the barrier is taken as $\Delta E_{c}=0.675 \Delta E_{g}$, since the reported values vary from $0.65 \Delta E_{g}$ to $0.7 \Delta E_{g}$ [24-26], where $\Delta E_{g}$ is the difference in band gap between the two semiconductors in the junction and is calculated as $25 \mathrm{meV} \%$ of $\mathrm{Mg}$ in the barrier. The effective masses of the QWs and the barriers are taken to be same as the $\mathrm{ZnO}$ polaron mass $(0.28$ $m_{0}$ ), due to the strong interaction between electrons and phonons in this highly ionic material, where $m_{0}$ is the free electron mass. The calculated band diagrams for all samples mainly consist of three energy levels, labeled $E_{0}, E_{1}$, and $E_{2}$, respectively. The Fermi level is indicated by $E_{F}$. ISBTs with different transition energies are expected for all the samples due to varied coupling strengths between the $\mathrm{ZnO}$ QWs.

In order to investigate the layer thicknesses and the heterointerface properties, the as-grown samples are characterized by high-angle annular dark-field imaging, scanning transmission electron microscopy, (STEM HAADF) and $\mathrm{x}$-ray reflectivity from which the thicknesses of the QWs and barriers can be obtained. Figure 2(a) is a STEM HAADF image of sample $A$, showing 20 periods of the 
ACQWs. The enlarged view of three periods is shown in Fig. 2(b). As reported before [27], the $\mathrm{ZnO} / \mathrm{Mg}_{x} Z_{1-x} \mathrm{O}$ QWs grown on nonpolar $m$ plane $\mathrm{ZnO}$ substrate feature a $\mathrm{V}$-shaped groove grating with orientation parallel to the $c$ axis. The same feature is also observed in Fig. 2(b). Despite this $\mathrm{V}$-shaped morphology, a homogeneous growth of the $\mathrm{Mg}_{0.3} \mathrm{Zn}_{0.7} \mathrm{O}$ barrier of 0.5 -nm thick can be readily recognized. The STEM HAADF images reveal an excellent structure and periodicity control and a good interface quality. To independently verify the growth quality, further material analysis is investigated by $\mathrm{x}$-ray reflectivity (XRR). Through comparison with the numerical simulation, several material properties can be derived, for instance, layer thickness (shown in Table I) and interface roughness. Figure 2(c) displays the experimental x-ray reflectivity spectra of all the investigated samples. Regularly spaced peaks can be observed for all samples. They represent constructive interferences from the sample periodicity. Thus, their angular positions can be used to measure the period thickness (i.e., $t_{\mathrm{QW} 1}+t_{B 1}+t_{\mathrm{QW} 2}+t_{B 2}$ ). Note that the change of sample periods is evidenced by the position shift of the reflectivity peaks. Sharp interference peaks can be easily observed, indicating an abrupt interface between QWs and barriers, a constant roughness between different layers, and a uniform thickness among different periods. However, this is not sufficient to independently determine the thicknesses $t_{\mathrm{QW} 1}, t_{B 1}, t_{\mathrm{QW} 2}$ and $t_{B 2}$ because $\mathrm{ZnO}$ and $(\mathrm{Zn}, \mathrm{Mg}) \mathrm{O}$ do not have identical growth rates. To do so, we use a calibration layer grown with the same growth conditions as the investigated samples. It consists of thick $\mathrm{ZnO}$ and $(\mathrm{Zn}, \mathrm{Mg}) \mathrm{O}$ layers from which we can deduce the $\mathrm{ZnO}$ and $(\mathrm{Zn}, \mathrm{Mg}) \mathrm{O}$ growth rates by scanning electron microscopy (SEM) measurements. By coupling the results of XRR and SEM, we are able to independently determine thicknesses with a precision of 1 monolayer (ML). Such high precision is important for accurate determination of the ISBTs in the ACQWs. As shown in Table I, the measured thickness shows excellent agreement with the designed values. For comparison, the simulated and experimental XRR spectra of sample $A$ are shown in Fig. 2(d), where a peak is missing at $2 \theta=1.94^{\circ}$. This peak extinction comes from a destructive interference. As for constructive interferences, this destructive interference originates from the sample periodicity. The simulation gives a QW thickness of $2.8 \mathrm{~nm}$ to explain this extinction, which is very close to the targeted thickness for QW2 $(2.5 \mathrm{~nm}$, see Table I), and in agreement with STEM thickness measurement if an error of $\pm 0.3 \mathrm{~nm}$ is considered. The structure surface morphology is also characterized with an atomic force microscopy (AFM). A typical AFM image of sample $A$, with a scan area of $10 \times 10 \mu \mathrm{m}^{2}$, is shown in the inset of Fig. 2(c), with a root-mean-square (RMS) surface roughness of $0.37 \mathrm{~nm}$, which is consistent with the value obtained from $\mathrm{x}$-ray reflectivity.
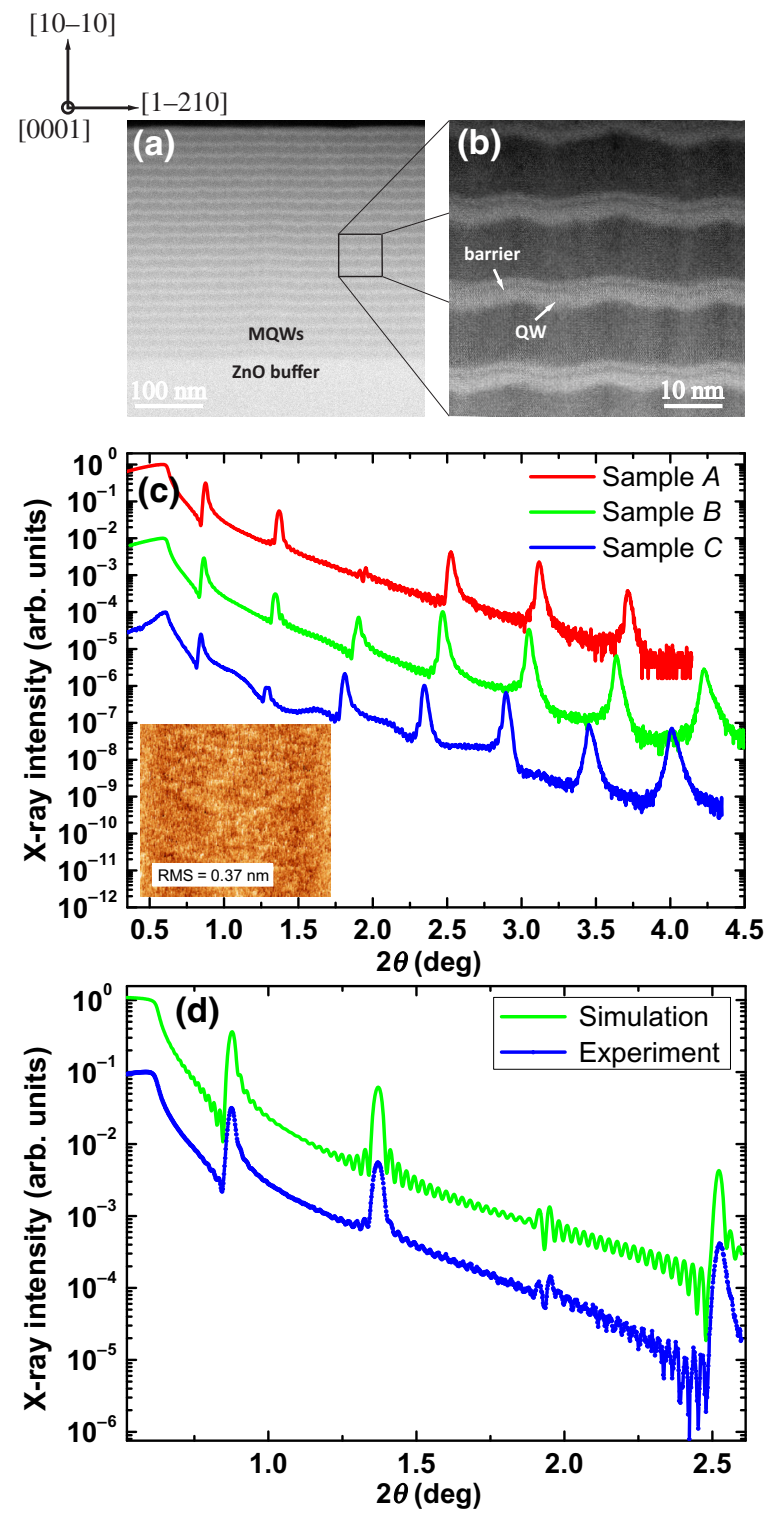

FIG. 2. (a) Cross-section HAADF STEM image of sample $A$ consisting of 20 periods of ACQWs. (b) Enlarged image of ACQWs of three periods, where the 0.5 -nm-thick $\mathrm{Mg}_{0.3} \mathrm{Zn}_{0.7} \mathrm{O}$ barrier is readily distinguishable. (c) X-ray reflectivity spectra for all the studied samples. The inset shows the AFM image of sample $A$, with a root-mean-square surface roughness of $0.37 \mathrm{~nm}$ in a $10 \times 10 \mu \mathrm{m}^{2}$ scan area. (d) Simulated and experimental XRR spectra of sample $A$.

\section{INTERSUBBAND ABSORPTION MEASUREMENTS AND DISCUSSION}

For infrared absorption measurements, the backsides of the samples are mechanically polished to optical quality with diamond paper of 500-nm-grain size. The transmission measurements of both TM and TE polarizations are performed at room temperature using fast scan in a Bruker Fourier transform infrared spectrometer (FTIR) 
with a deuterated triglycine sulfate (DTGS) photodetector. A KRS-5 holographic wire-grid polarizer is inserted in the optical path to select the TM or TE polarizations of the incident light on the samples. The absorption is measured using the single-pass Brewster's angle geometry and a home-built holder, with an incident angle of approximately $62^{\circ}\left(n_{\mathrm{ZnO}} \sim 1.9\right.$ at $\left.\lambda \sim 3 \mu \mathrm{m}\right)$. As the $c$ axis lies in the plane of QWs, to suppress the anisotropic effect of the material, the TM polarization is chosen to have an electric field perpendicular to the $c$ axis, whereas the TE polarization has an electric field horizontal to the $c$ axis (see Supplemental Material [28]). To subtract the polarization dependence of the optics in the spectrometer, two background transmission spectra $\left(T_{\mathrm{TE}}^{\text {back }}, T_{\mathrm{TM}}^{\text {back }}\right)$ are also recorded. Together with the two transmission spectra with samples $\left(T_{\mathrm{TE}}^{\text {sample }}, T_{\mathrm{TM}}^{\text {sample }}\right.$ ), the absorption can be calculated as

$$
\text { Absorption }=-\ln \left(T_{\mathrm{TM}}^{\text {sample }} / T_{\mathrm{TM}}^{\text {back }}\right)+\ln \left(T_{\mathrm{TE}}^{\text {sample }} / T_{\mathrm{TE}}^{\text {back }}\right) \text {. }
$$

Figure 3 displays the normalized absorption for three investigated samples at room temperature. Absorption signals are visible from approximately $1200 \mathrm{~cm}^{-1}$ to approximately $4800 \mathrm{~cm}^{-1}$. Compared with the single QW case, which exhibits a single absorption peak [21,24,29], two absorption peaks are distinguishable for all the samples. The absorption spectra show a strong TM polarization dependence, thus demonstrating the same selection rule for intersubband transitions as in a single QW structure [21]. The absorption below $1200 \mathrm{~cm}^{-1}$ is due to the multiphonon absorption of the $m$ plane $\mathrm{ZnO}$ substrate. Layer thicknesses deduced from the $\mathrm{x}$-ray reflectivity method are used in the calculation. As in the case of a single QW, a significant change of the transition energy due to the depolarization shift is also considered because of the high doping. The charge-induced coherence due to the strong dipole-dipole Coulomb interaction between ISBTs cannot be ignored due to the high doping level [30,31], thus leading to the formation of multisubband plasmons (Supplemental Material [28]). To take into account the charge-induced coherence effect, a complete quantum model in Ref. [31] based on the dipole representation is used to explain the observed absorption spectra. In the calculation, each multisubband plasmon transition is assumed to have a Gaussian line shape due to the long-range interface fluctuation and the heavy mass of the $\mathrm{ZnO}$ quantum well, with the line width as a fitting parameter. In the calculation, the half width at half maximum (HWHM) of for all the transitions is kept at $560 \mathrm{~cm}^{-1}$, which is calculated by considering the interface roughness as the main broadening mechanism and taking the multiple QW ZnO absorption as a reference (e.g., HWHM is $420 \mathrm{~cm}^{-1}$ in this case). However, it should be noted that the line width of the low energy absorption may be underestimated in the calculation. To accurately locate

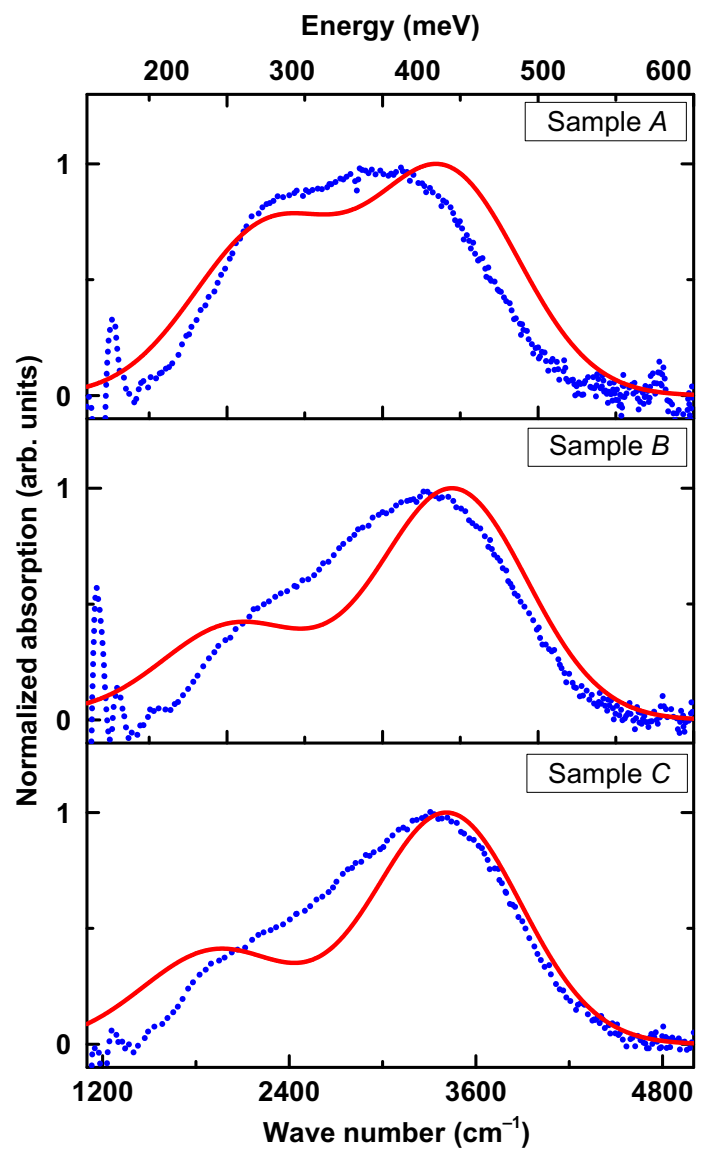

FIG. 3. Intersubband absorption spectra (blue dotted line) for all the samples in the study with FTIR. The spectra are calibrated using Eq. (1). The red solid lines show the corresponding calculated spectra.

the transition energies of the intersubband absorption, the absorption spectra are fitted with a sum of two Gaussian peaks, with peak energy and the FWHM as the free parameters. The experimental peak energies and intensity ratios obtained using the above process are compared with the numerical values in Fig. 4, where the inset shows the comparison of the absorption spectrum of sample $A$ and two fitting Gaussian spectra. Though a more advanced numerical simulation method provides a better description of the carrier dynamics in complex quantum structure, the simplified model used here shows reasonable overall agreement with the experimental results, given the extreme sensitivity of the ISBTs on the ACQWs parameters. In the absorption spectra, two main peaks can be easily distinguished, which are located at approximately $2000 \mathrm{~cm}^{-1}$ and approximately $3500 \mathrm{~cm}^{-1}$, respectively. Physically, in the multisubband plasmon picture, the low energy peak mainly consists of transition $E_{01}=E_{1}-E_{0}$, whereas the high energy peak mainly consists of transition $E_{02}=E_{2}-E_{0}$. As the barrier thickness increases from 0.5 to $1.5 \mathrm{~nm}$, the coupling between the two QWs decreases. 
(a)
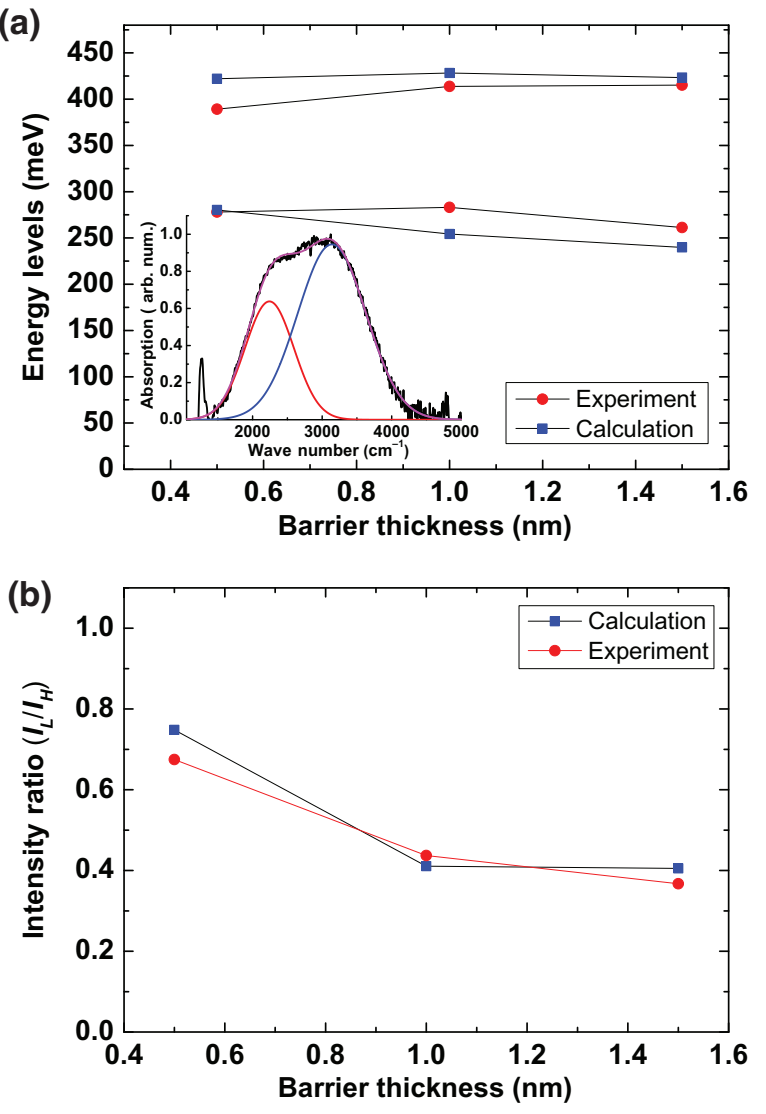

FIG. 4. (a) Experimental (red disk) and calculated (blue rectangle) intersubband absorption peaks for all the samples. The inset shows the comparison of the experimental absorption of sample $A$ and two Gaussian fitting functions. (b) Experimental and calculated intensity ratios for all the samples.

Accordingly, in the single particle picture, it is shown that the ratio between oscillator strengths $f_{01} / f_{02}$ is calculated to decrease by a factor of approximately 10 , where $f_{01}$ and $f_{02}$ correspond to the oscillator strengths of transitions $E_{01}$ and $E_{02}$, respectively. As a result, the calculated absorption ratio $R=A_{L} / A_{H}$, where $A_{L}$ and $A_{H}$ correspond to the absorption amplitudes of observed low- and highenergy multisubband plasmons, respectively, decreases from 0.75 to 0.41 , which is in reasonable agreement with the experimental value ( 0.67 to 0.37 ) obtained by comparing the amplitudes of two fitting Gaussian functions [in Fig. 4(b)]. We notice that the fitted HWHM of the ISBTs absorption is in the range of approximately $420 \mathrm{~cm}^{-1}$ to approximately $600 \mathrm{~cm}^{-1}$, which is larger than the measured value in the multiple QW with similar doping levels (HWHM $\sim 400 \mathrm{~cm}^{-1}$ in the $m$ plane) [21]. We attribute this to the enhanced detrimental impact of interface roughness from the tunneling barrier. As the wave functions exhibit large amplitudes at the interface, stronger scattering is expected, thus leading to a broader line width. The absorption line-width broadening has also been observed in the ACQWs based on a GaN/AIN material system [22], which shows line widths to 2-3 times of the state-of-art single QW value.

\section{CONCLUSION}

In conclusion, we report the direct observation of intersubband optical transitions in ACQWs based on a $\mathrm{ZnO} / \mathrm{Mg}_{0.3} \mathrm{Zn}_{0.7} \mathrm{O}$ material system grown by MBE on nonpolar $m$ plane substrates. The grown ACQWs structures are systematically characterized, assuring excellent material quality for ISBT-based studies, with a thickness control below one monolayer. Using the Brewster's angle absorption geometry, the strong coupling between two QWs is evidenced by the observation of two intersubband absorption peaks. The absorption peaks feature broader line widths than the single QW value, resulting from the more pronounced interface roughness scattering in the ACQWs. Using a simple Schrödinger-Poisson solver and a complete quantum model including interaction between different intersubband transitions, the theoretical simulation provides reasonable agreement with the experimental results, which unambiguously demonstrates the strong coupling between the two quantum wells. The discrepancy between the theory and experiment may be attributed to the uncertainties of the material parameters (e.g., effective mass and band offset), the fluctuation of the layer thickness, that is, ML variation, and the $V$ groove shape of the heterostructures. The investigation of the impact of the $\mathrm{V}$ groove shape on the absorption is now underway. These results show a step forward in the realization of $\mathrm{ZnO} / \mathrm{Mg}_{\mathrm{x}} \mathrm{Zn}_{1-\mathrm{x}} \mathrm{O}$ optoelectronic devices based on ISBTs, especially $\mathrm{THz}$ QCLs.

\section{ACKNOWLEDGMENTS}

Part of the calculations were performed use the Aestimo solver [32]. We thank the National Center for Electron Microscopy (CNME; UCM, Madrid) and its director, Professor José M. González-Calbet, for facilities. This work is funded by the European Union's Horizon 2020 Research and Innovation Program under Grant No. 665107 (project ZOTERAC).

[1] Atsushi Tsukazaki, Masashi Kubota, Akira Ohtomo, Takeyoshi Onuma, Keita Ohtani, Hideo Ohno, Shigefusa F. Chichibu, and Masashi Kawasaki, Blue light-emitting diode based on ZnO, Jpn. J. Appl. Phys. 44, L643 (2005).

[2] M. Willander, et al., Zinc oxide nanorod based photonic devices: recent progress in growth, light emitting diodes and lasers, Nanotechnology 20, 332001 (2009).

[3] Yungryel Ryu, Tae-Seok Lee, Jorge A. Lubguban, Henry W. White, Bong-Jin Kim, Yoon-Soo Park, and Chang-Joo 
Youn, Next generation of oxide photonic devices: $\mathrm{ZnO}-$ based ultraviolet light emitting diodes, Appl. Phys. Lett. 88, 241108 (2006).

[4] Marian Zamfirescu, Alexey Kavokin, Bernard Gil, Guillaume Malpuech, and Mikhail Kaliteevski, ZnO as a material mostly adapted for the realization of room-temperature polariton lasers, Phys. Rev. B 65, 161205 (2002).

[5] R. Shimada, J. Xie, V. Avrutin, Ü Özgür, and H. Morkoč, Cavity polaritons in $\mathrm{ZnO}$-based hybrid microcavities, Appl. Phys. Lett. 92, 011127 (2008).

[6] Lambert K. van Vugt, Sven Rühle, Prasanth Ravindran, Hans C. Gerritsen, Laurens Kuipers, and Daniël Vanmaekelbergh, Exciton Polaritons Confined in a $\mathrm{ZnO}$ Nanowire Cavity, Phys. Rev. Lett. 97, 147401 (2006).

[7] A. Tsukazaki, A. Ohtomo, T. Kita, Y. Ohno, H. Ohno, and M. Kawasaki, Quantum hall effect in polar oxide heterostructures, Science 315, 1388 (2007).

[8] J. Falson, D. Maryenko, B. Friess, D. Zhang, Y. Kozuka, A. Tsukazaki, J. H. Smet, and M. Kawasaki, Evendenominator fractional quantum Hall physics in $\mathrm{ZnO}$, Nat. Phys. 11, 347 (2015).

[9] A. Tsukazaki, S. Akasaka, K. Nakahara, Y. Ohno, H. Ohno, D. Maryenko, A. Ohtomo, and M. Kawasaki, Observation of the fractional quantum Hall effect in an oxide, Nat. Mater. 9, 889 (2010).

[10] W. S. Baer, Faraday rotation in ZnO: Determination of the electron effective mass, Phys. Rev. 154, 785 (1967).

[11] A. Jollivet, B. Hinkov, S. Pirotta, H. Hoang, S. Derelle, J. Jaeck, M. Tchernycheva, R. Colombelli, A. Bousseksou, M. Hugues, N. Le Biavan, J. Tamayo-Arriola, M. Montes Bajo, L. Rigutti, A. Hierro, G. Strasser, J.-M. Chauveau, and F. H. Julien, Short infrared wavelength quantum cascade detectors based on m-plane $\mathrm{ZnO} / \mathrm{ZnMgO}$ quantum wells, Appl. Phys. Lett. 113, 251104 (2018).

[12] Benedikt Schwarz, Daniela Ristanic, Peter Reininger, Tobias Zederbauer, Donald MacFarland, Hermann Detz, Aaron Maxwell Andrews, Werner Schrenk, and Gottfried Strasser, High performance bi-functional quantum cascade laser and detector, Appl. Phys. Lett. 107, 071104 (2015).

[13] Keita Ohtani, Bo Meng, Martin Franckié, Lorenzo Bosco, Camille Ndebeka-Bandou, Mattias Beck, and Jérôme Faist, An electrically pumped phonon-polariton laser, Sci. Adv. 5, eaau1632 (2019).

[14] Jerome Faist, Federico Capasso, Deborah L. Sivco, Carlo Sirtori, Albert L. Hutchinson, and Alfred Y. Cho, Quantum cascade laser, Science 264, 553 (1994).

[15] Rüdeger Köhler, Alessandro Tredicucci, Fabio Beltram, Harvey E. Beere, Edmund H. Linfield, A. Giles Davies, David A. Ritchie, Rita C. Iotti, and Fausto Rossi, Terahertz semiconductor-heterostructure laser, Nature 417, 156 (2002).

[16] V. P. Sirkeli and H. L. Hartnagel, ZnO-based terahertz quantum cascade lasers, Opto-Electron. Rev. 27, 119 (2019).

[17] Enrico Bellotti and Roberto Paiella, Numerical simulation of $\mathrm{ZnO}$-based terahertz quantum cascade lasers, J. Electron. Mater. 39, 1097 (2010).

[18] N. Le Biavan, M. Hugues, M. Montes Bajo, J. TamayoArriola, A. Jollivet, D. Lefebvre, Y. Cordier, B. Vinter, F.-H. Julien, A. Hierro, and J.-M. Chauveau, Homoepitaxy of non-polar $\mathrm{ZnO} /(\mathrm{Zn}, \mathrm{Mg}) \mathrm{O}$ multi-quantum wells: From a precise growth control to the observation of intersubband transitions, Appl. Phys. Lett. 111, 231903 (2017).

[19] Mattias Beck, Daniel Hofstetter, Thierry Aellen, Jérôme Faist, Ursula Oesterle, Marc Ilegems, Emilio Gini, and Hans Melchior, Continuous wave operation of a midinfrared semiconductor laser at room temperature, Science 295, 301 (2002).

[20] J.-M. Chauveau, M. Teisseire, H. Kim-Chauveau, C. Deparis, C. Morhain, and B. Vinter, Benefits of homoepitaxy on the properties of nonpolar $(\mathrm{Zn}, \mathrm{Mg}) \mathrm{O} / \mathrm{ZnO}$ quantum wells on a-plane $\mathrm{ZnO}$ substrates, Appl. Phys. Lett. 97, 081903 (2010).

[21] Miguel Montes Bajo, Julen Tamayo-Arriola, Maxime Hugues, Jose M. Ulloa, Nolwenn Le Biavan, Romain Peretti, Francois H. Julien, Jerome Faist, Jean-Michel Chauveau, and Adrian Hierro, Multisubband Plasmons in Doped ZnO Quantum Wells, Phys. Rev. Appl. 10, 024005 (2018).

[22] M. Tchernycheva, L. Nevou, L. Doyennette, F. H. Julien, F. Guillot, E. Monroy, T. Remmele, and M. Albrecht, Electron confinement in strongly coupled GaNấţAlN quantum wells, Appl. Phys. Lett. 88, 153113 (2006).

[23] Walter L. Bloss, Effects of Hartree, exchange, and correlation energy on intersubband transitions, J. Appl. Phys. 66, 3639 (1989).

[24] M. Belmoubarik, K. Ohtani, and H. Ohno, Intersubband transitions in $\mathrm{ZnO}$ multiple quantum wells, Appl. Phys. Lett. 92, 191906 (2008).

[25] Kuaile Zhao, Guopeng Chen, Bing-Sheng Li, and Aidong Shen, Mid-infrared intersubband absorptions in $\mathrm{ZnO} / \mathrm{ZnMgO}$ multiple quantum wells, Appl. Phys. Lett. 104, 212104 (2014).

[26] Haitao Yin, Junli Chen, Yin Wang, Jian Wang, and Hong Guo, Composition dependent band offsets of $\mathrm{ZnO}$ and its ternary alloys, Sci. Rep. 7, 41567 (2017).

[27] E. Di Russo, L. Mancini, F. Moyon, S. Moldovan, J. Houard, F. H. Julien, M. Tchernycheva, J. M. Chauveau, M. Hugues, G. Da Costa, I. Blum, W. Lefebvre, D. Blavette, and L. Rigutti, Three-dimensional atomic-scale investigation of $\mathrm{ZnO}-\mathrm{Mg}_{x} \mathrm{Zn}_{1-x} \mathrm{O} m$-plane heterostructures, Appl. Phys. Lett. 111, 032108 (2017).

[28] See Supplemental Material http://link.aps.org/supplemen tal/10.1103/PhysRevApplied.12.054007 for more information on the single-pass Brewster angle geometry and the comparison of absorption spectra using the single particle and the multisubband plasmon pictures.

[29] K. Ohtani, M. Belmoubarik, and H. Ohno, Intersubband optical transitions in $\mathrm{ZnO}$-based quantum wells grown by plasma-assisted molecular beam epitaxy, J. Crystal Growth 311, 2176 (2009).

[30] A. Delteil, A. Vasanelli, Y. Todorov, C. Feuillet Palma, M. Renaudat St-Jean, G. Beaudoin, I. Sagnes, and C. Sirtori, Charge-Induced Coherence between Intersubband Plasmons in a Quantum Structure, Phys. Rev. Lett. 109, 246808 (2012).

[31] G. Pegolotti, A. Vasanelli, Y. Todorov, and C. Sirtori, Quantum model of coupled intersubband plasmons, Phys. Rev. B 90, 035305 (2014).

[32] The solver can be found at http://www.aestimosolver.org/. 